The Astrophysical Journal, 593:L27-L30, 2003 August 10

(C) 2003. The American Astronomical Society. All rights reserved. Printed in U.S.A.

\title{
X-RAY OBSERVATIONS OF THE COMPACT CENTRAL OBJECT IN SUPERNOVA REMNANT G347.3-0.5
}

\author{
J. S. Lazendic, ${ }^{1}$ P. O. Slane, ${ }^{1}$ B. M. Gaensler, ${ }^{1}$ P. P. Plucinsky, ${ }^{1}$ J. P. Hughes, ${ }^{2}$ D. K. Galloway, ${ }^{3}$ and F. Crawford ${ }^{4}$ \\ Received 2003 June 2; accepted 2003 July 3; published 2003 July 15
}

\begin{abstract}
We present Chandra, XMM-Newton, and Rossi X-Ray Timing Explorer observations of 1WGA J1713.4-3949, a compact source at the center of the Galactic supernova remnant (SNR) G347.3-0.5. The X-ray spectrum of the source is well fitted by the sum of a blackbody component with a temperature of $\sim 0.4 \mathrm{keV}$ plus a power-law component with a photon index of $\sim 4$. We found no pulsations down to $4 \%$ in the $0.01-0.16 \mathrm{~Hz}$ range nor down to $25 \%$ in the $0.01-128 \mathrm{~Hz}$ range. This source resembles other compact central objects in SNRs, and we suggest that 1WGA J1713.4-3949 is the associated neutron star for G347.3-0.5. We also measured the properties of the adjacent radio pulsar PSR J1713-3945 with a $392 \mathrm{~ms}$ period, and we show that it is not associated with 1 WGA J1713.4-3949 nor, most probably, is it associated with SNR G347.3-0.5.
\end{abstract}

Subject headings: ISM: individual (G347.3-0.5) — pulsars: individual (PSR J1713-3945) — stars: neutron — supernova remnants - X-rays: stars

\section{INTRODUCTION}

For many years, it has been well known that pulsars-the compact remains of supernova explosions - are rapidly rotating, highly magnetized neutron stars (NSs). However, sources discovered in recent years have shown that NSs also appear to come in other varieties: even more highly magnetized objects with slow spins, such as anomalous X-ray pulsars and soft gamma-ray repeaters (e.g., Mereghetti 1998; Thompson 2000), and more poorly understood objects near the centers of supernova remnants (SNRs) with detectable X-ray flux but no optical/radio counterparts nor any signs of rotation, so-called compact central objects (CCOs; Pavlov et al. 2002). Their spectra are very soft, with blackbody temperatures of $\sim 0.4 \mathrm{keV}$, often requiring an additional power-law component with photon indices of $\sim 4$ (Pavlov et al. 2002). In this Letter, we address the nature of 1WGA J1713.4-3949, a compact source located at the center of the SNR G347.3-0.5 (RX J1713.7-3946). Early ROSAT observations identified two point sources within the boundaries of the SNR shell (Pfeffermann \& Aschenbach 1996; see also Fig. 1). The source farthest from the center of the remnant, 1WGA J1714.4-3945, was determined to be of stellar origin (Pfeffermann \& Aschenbach 1996). For the other source, 1WGA J1713.4-3949, located at the geometrical center of the SNR, no optical counterpart has been found within $10^{\prime \prime}$ of the ROSAT position (Slane et al. 1999), and the source has correspondingly been suggested to be a candidate for an associated NS. While ASCA observations were not able to provide useful limits to the pulsations in the X-ray band (Slane et al. 1999), radio pulsations were detected with a $392 \mathrm{~ms}$ period within a $7^{\prime}$ radius region toward this source (PSR J1713-3945; Crawford et al. 2002).

1WGA J1713.4-3949 has been observed with the Chandra $X$-Ray Observatory as part of a project to study nonthermal radio and X-ray emission from the SNR shell (Lazendic et al. 2003). In this Letter, we present a detailed spectral and timing

\footnotetext{
${ }^{1}$ Harvard-Smithsonian Center for Astrophysics, 60 Garden Street, Cambridge, MA 02138; jlazendic@cfa.harvard.edu.

${ }^{2}$ Department of Physics and Astronomy, Rutgers University, 136 Frelinghuysen Road, Piscataway, NJ 08854.

${ }^{3}$ Center for Space Research, Massachusetts Institute of Technology, 70 Vassar Street, Building 37, Cambridge, MA 02139.

${ }^{4}$ Department of Physics and Astronomy, Haverford College, Haverford, PA 19041.
}

analysis of 1WGA J1713.4-3949 using our Chandra data as well as archival data from the XMM-Newton and Rossi X-Ray Timing Explorer (RXTE) satellites. We also present properties of the radio pulsar PSR J1713-3945 that imply that it is not related to 1WGA J1713.4-3949 or SNR G347.3-0.5.

\section{OBSERVATIONS}

Two fields toward G347.3-0.5 were observed on 2000 July 25 with the Advanced CCD Imaging Spectrometer (ACIS) detector on board the Chandra X-Ray Observatory (observation ID [ObsID] 736 and 737). The ACIS-I detector for ObsID 736 was positioned at the bright northwestern rim of the SNR, and the roll angle was such that 1WGA J1713.4-3949 fell on the S1 chip (see Fig. 1). Data were taken in timed exposure mode with an integration time of $3.2 \mathrm{~s}$ per frame. The fully processed level 2 data were reduced using standard threads in the CIAO software package, version 2.2.1. The effective exposure time after data processing was $29.6 \mathrm{ks}$. The exposure-corrected Chandra image of the SNR and the compact source 1WGA J1713.4-3949 is shown in Figure 1, with the ROSAT contours overlaid. The image was binned in 4".6 $\times 4$ ".6 pixels and smoothed with a Gaussian filter with an FWHM of $2^{\prime \prime}$. The image of the compact source is elongated because its location is well off-axis $\left(\sim 23^{\prime}\right)$ from the aim point.

We obtained archival XMM data of 1WGA J1713.4-3949 carried out on 2001 March 2 with a 15 ks exposure (ObsID 0093670501; G. Cassam-Chenai, A. Decourchelle, J. Ballet, J.-L. Sauvageot, \& G. Dubner 2003, in preparation). The European Photon Imaging Camera (EPIC) pn camera was operated in extended full frame mode with a time resolution of $199 \mathrm{~ms}$, while the two EPIC-MOS cameras were operated in full frame mode with a time resolution of $2.6 \mathrm{~s}$. The EPIC data reduction was performed using the SAS software package, version 5.4.1. The event files used for analysis were created from observational data files using the SAS tasks EPCHAIN and EMCHAIN. The net exposure time after filtering event files for good time intervals was $9.2 \mathrm{ks}$ in the pn camera and $14.1 \mathrm{ks}$ in each MOS camera.

We used archival data of SNR G347.3-0.5 (Pannuti et al. 2003) from the Proportional Counter Array (PCA) aboard the RXTE satellite; the PCA is sensitive to X-ray photons in the 2-60 keV energy band and has a field of view with a radius of $\approx 1^{\circ}$. While the PCA has no imaging capability, it has a large effective area $\left(6000 \mathrm{~cm}^{2}\right)$ that makes it suitable for searching 


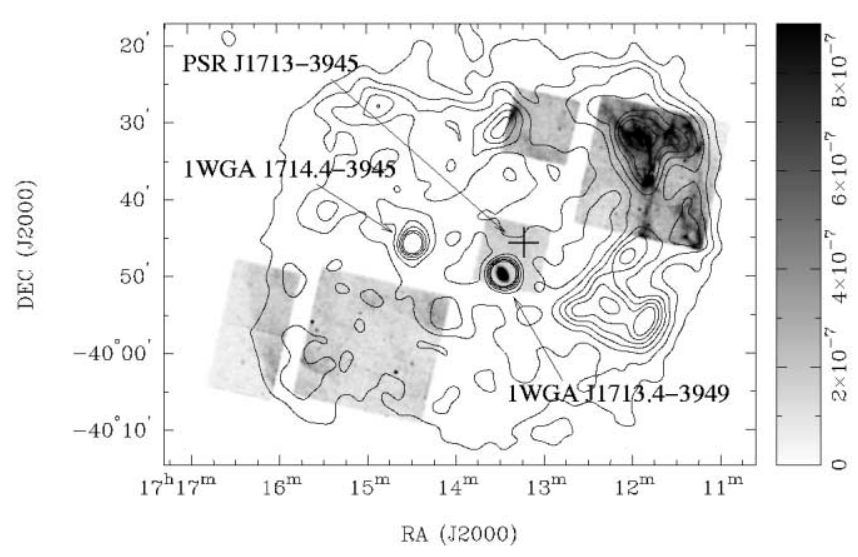

FIg. 1.-The $0.5-10.0 \mathrm{keV}$ band Chandra gray-scale image of the two fields observed toward the SNR G347.3-0.5 (see Lazendic et al. 2003 for more details). To show the whole extent of the remnant, the Chandra image is overlaid with the ROSAT PSPC contours (Slane et al. 1999). The two point sources that were detected with ROSAT as well as the location of the radio pulsar PSR J1713-3945 are labeled.

for faint X-ray pulsations. Observations of G347.3-0.5 were made in seven separate intervals between 1999 June 14 and 24 , with a total exposure time of $100 \mathrm{ks}$. Analysis of the RXTE data was carried out using LHEASOFT software, version 5.2.

A $392 \mathrm{~ms}$ radio pulsar, PSR J1713-3945, was discovered in a $20 \mathrm{~cm}$ targeted search for pulsations in SNR G347.3-0.5 using the Parkes telescope and multibeam receiver. The details of the search and discovery are outlined in Crawford et al. (2002). The pulsar was subsequently timed with the multibeam receiver at Parkes to obtain astrometric and spin parameters and to determine whether the pulsar was associated with 1WGA J1713.4-3949.

\section{ANALYSIS AND RESULTS}

\subsection{Spectral Analysis}

For the spectral analysis of the Chandra data, we extracted the source counts from a $37^{\prime \prime} \times 61^{\prime \prime}$ elliptical region around the compact source, and for the background region, we used a $2^{\prime} \times 3^{\prime}$ elliptical region north of the source. We obtained $8454 \pm 103$ background-subtracted counts for the source, and the spectrum was grouped to contain at least 25 counts bin ${ }^{-1}$. Residuals from fitting standard continuum models to the source spectrum (blackbody, bremsstrahlung, and power law) revealed an apparent change in the gain calibration on chip S1 that results in a spurious absorption feature around $2 \mathrm{keV}$. We extracted data from the on-board calibration source taken before and after our observation, and these show that there is indeed a gain shift for this CCD of $\sim 3.5 \%$. However, we found that the fit parameters did not change significantly when the gain was frozen at this $3.5 \%$ value or when it was left as a free parameter. We thus allowed gain to vary in our models (see Table 1).

For the spectral analysis of the $X M M$ data, we obtained $9600 \pm 217$ background-subtracted counts from the pn camera, and $5451 \pm 74(5274 \pm 73)$ from the MOS1 (MOS2) camera. In the pn camera, the counts were extracted from a 27 " radius circular region around the source, and for the background extraction, we used a 3'.4 × 8.3 box north from the source position inside the same CCD chip. Similarly, in the MOS1 (MOS2) camera, we used a 22" (19") radius circular region for the source and a $3.4(2 ! 2)$ radius circular region on the same chip for the background. The spectra were also grouped to contain at least 25 counts bin ${ }^{-1}$. We performed a joint spectral analysis of the three EPIC data sets, allowing only the relative normalization to vary. The results obtained were consistent with those obtained from the Chandra spectrum. We therefore proceeded with a joint spectral analysis of Chandra and XMM spectra.

The results from fitting a few typical NS models are summarized in Table 1, and a fit example is given in Figure 2. Fits with the bremsstrahlung and blackbody models gave equally acceptable results, while the power-law model gave a somewhat higher reduced $\chi^{2}$. The steep photon index of $\Gamma \sim 4$ excludes the possibility that 1WGA J1713.4-3949 is a background active galactic nucleus because active galactic nuclei typically have photon indices of 1.2-2.2 (Turner \& Pounds 1989). The best-fit column density $\left(N_{\mathrm{H}}\right)$ of $\sim 11 \times 10^{21} \mathrm{~cm}^{-2}$ in the power-law model is also somewhat higher than the value of $8 \times 10^{21} \mathrm{~cm}^{-2}$ found toward the SNR (Lazendic et al. 2003). On the other hand, a blackbody model yields $N_{\mathrm{H}} \sim 4 \times 10^{21} \mathrm{~cm}^{-2}$, which is a lower value than that found toward the SNR. The blackbody fit is improved by adding a power-law component, which also results in an $N_{\mathrm{H}}$ value consistent with that found toward the SNR. In this composite spectrum, the unabsorbed source luminosity is $L_{\mathrm{X}}(0.5-10.0 \mathrm{keV}) \sim 6 \times 10^{34} \mathrm{erg} \mathrm{s}^{-1}$, adopting a distance of $\sim 6 \mathrm{kpc}$ to the SNR (Slane et al. 1999). The blackbody normalization yields a relatively small emitting area with radius $R \sim$ $2.4 D_{6 \mathrm{kpc}} \mathrm{km}$, which is significantly smaller than the canonical value for the NS radius of $10 \mathrm{~km}$. This could imply that most of the thermal emission originates from the polar caps on the NS surface; the thermal component from the rest of the NS surface is too faint to be detected.

The small inferred radius may be at least partly due to the

TABLE 1

Parameters of Joint Spectral Fits to ChandRa and XMM Spectra of 1WGA J1713.4-3949 with DIFFERENT MODELS

\begin{tabular}{|c|c|c|c|c|c|c|c|}
\hline Model $^{\mathrm{a}}$ & $\begin{array}{c}N_{\mathrm{H}} \\
\left(\times 10^{21} \mathrm{~cm}^{-2}\right)\end{array}$ & $\begin{array}{c}k T \\
(\mathrm{keV})\end{array}$ & $\Gamma$ & $\begin{array}{l}R_{\infty}{ }^{\mathrm{b}} \\
(\mathrm{km})\end{array}$ & $\chi_{\mathrm{red}}^{2} / \mathrm{dof}$ & $\begin{array}{c}\operatorname{Gain}^{\mathrm{c}} \\
(\%)\end{array}$ & $\begin{array}{c}F_{\mathrm{X}}^{0}(0.5-10.0 \mathrm{keV})^{\mathrm{d}} \\
\left(\operatorname{ergs~cm}^{-2} \mathrm{~s}^{-1}\right)\end{array}$ \\
\hline BREMSS & $7.0_{-0.2}^{+0.1}$ & $0.92_{-0.02}^{+0.02}$ & & & $1.07 / 675$ & $5.1_{-0.2}^{+0.5}$ & $9.4 \times 10^{-12}$ \\
\hline POW. & $7_{-02}^{+0.4}$ & & $4.2_{-01}^{+0.1}$ & & $1.44 / 675$ & $4.6_{-05}^{+0.8}$ & $3.2 \times 10^{-11}$ \\
\hline BB .. & $3.6_{-0.1}^{+0.1}$ & $0.40_{-0.01}^{+0.01}$ & & $2.5_{-0.1}^{+0.1}$ & $1.25 / 675$ & $6.0_{-0.6}^{+0.5}$ & $4.5 \times 10^{-12}$ \\
\hline $\mathrm{POW}+\mathrm{BB} \ldots \ldots$ & $8.0_{-0.6}^{+0.9}$ & $0.38_{-0.02}^{+0.01}$ & $3.9_{-0.2}^{+0.3}$ & $2.4_{-0.4}^{+0.6}$ & $1.05 / 670$ & $7.1_{-0.8}^{+0.9}$ & $1.4(0.3) \times 10^{-11}$ \\
\hline $\mathrm{POW}+\mathrm{NSA}(\mathrm{G}) \ldots \ldots$ & & $0.18_{-0}^{+0}$ & $3.7_{-0.3}^{+0.8}$ & $13.0_{-3.5}^{+1.0}$ & $1.07 / 670$ & $5.5_{-0.5}^{+0.6}$ & $1.1(0.4) \times 10^{-11}$ \\
\hline $\mathrm{POW}+\mathrm{NSA}(\mathrm{Z}) \ldots$. & $8.1_{-0.9}^{+1.1}$ & $0.26_{-0.06}^{+0.03}$ & $3.8_{-0.5}^{+0.2}$ & $15.8_{-8.4}^{+4.2}$ & $1.22 / 667$ & $5.1_{-0.5}^{+0.5}$ & $1.5(0.3) \times 10^{-11}$ \\
\hline
\end{tabular}

${ }^{a}$ BREMSS = bremsstrahlung model; POW = power-law model; BB = blackbody model; NSA(G) $=$ NSA model by Gänsicke, Braje, \& Romani 2002 for a weak magnetic field of $\leq 10^{10} \mathrm{G}$; and NSA(Z) = NSA model by Pavlov et al. 1995 for a standard magnetic field of $10^{12} \mathrm{G}$.

${ }^{\mathrm{b}}$ Radius of the emitting region at infinity assuming the source distance of $6 \mathrm{kpc}$.

${ }^{\mathrm{c}}$ A linear gain shift included for fitting the Chandra data.

${ }^{\mathrm{d}}$ Unabsorbed flux; for the two-component models, the flux of the second component alone is given in parentheses. 


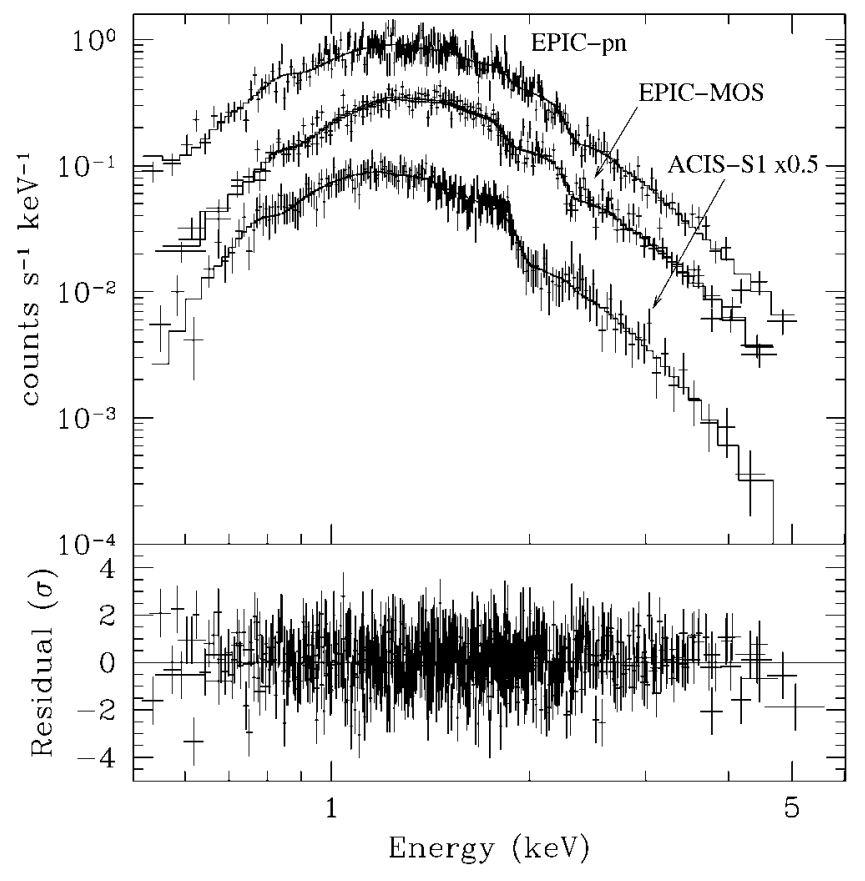

FIG. 2.-ACIS and EPIC spectra from the compact source 1WGA J1713.4-3949 and residuals for the blackbody+power-law model. The fit parameters are listed in Table 1.

oversimplification of the thermal emission from the NS by the blackbody model. More appropriate models that take into account the NS atmosphere (NSA) yield lower NS temperatures, which in turn correspond to larger NS radii (e.g., Zavlin, Pavlov, \& Shibanov 1996). To investigate this, we fitted our spectra with two NSA models. The NSA model by Gänsicke et al. (2002) is calculated for a weakly magnetic NS $\left(B \leq 10^{10} \mathrm{G}\right)$ and considers hydrogen, solar, or iron compositions of the atmosphere. The NSA model by Pavlov et al. (1995) assumes the standard magnetic field strength $\left(B=10^{12} \mathrm{G}\right)$ and a hydrogen atmosphere. Both models assume a standard NS mass of $1.4 M_{\odot}$. Both hydrogen models gave acceptable residuals and yielded emitting region sizes close to $10 \mathrm{~km}$, with corresponding reductions in the NS temperature. For either model, however, the derived temperature is above the expected surface temperature for a cooling NS more than several hundred years old. Model atmospheres with solar abundance or an iron composition gave unacceptable fits predicting broad absorption features not present in the spectra.

\subsection{Timing Analysis}

We used a fast Fourier transform (FFT) to search the X-ray data for pulsations from 1WGA J1713.4-3949 but found no pulsed signal; we list upper limits on the pulsed fraction and the search parameters in Table 2. The upper limit from RXTE data is least constraining because 1WGA J1713.4-3949 only accounts for $0.6 \%$ of the total flux in the RXTE's large field of view.

To derive the timing properties of the radio pulsar PSR J1713-3945, the TEMPO timing package was used on a total of 27 times of arrival (TOAs) with the JPL DE200 planetary ephemeris (Standish 1990). The TEMPO package converts each TOA to the solar system barycenter and then refines the timing parameters by minimizing residuals between observed and model TOAs over the observation span. The resulting timing parameters are presented in Table 3. The pulsar's dispersion
TABLE 2

Parameters and Results of Pulsation Search from 1WGA J1713.4-3949 Using FFT

\begin{tabular}{|c|c|c|c|}
\hline Detector & Time Resolution & $\begin{array}{c}\text { Frequency Range } \\
\text { Searched } \\
(\mathrm{Hz})\end{array}$ & $\begin{array}{l}f_{p} \text { Limit }^{\mathrm{a}} \\
(\%)\end{array}$ \\
\hline ACIS-S1 $\ldots$ & $3.2 \mathrm{~s}$ & $0.01-0.16$ & 12 \\
\hline EPIC-MOS $^{\mathrm{b}} \ldots \ldots$ & $2.6 \mathrm{~s}$ & $0.01-0.19$ & 12 \\
\hline EPIC-pn .......... & $199 \mathrm{~ms}$ & $0.01-2.5$ & 4 \\
\hline $\mathrm{PCA}^{\mathrm{c}} \ldots \ldots \ldots \ldots$ & $4 \mathrm{~ms}$ & $0.01-128$ & 25 \\
\hline
\end{tabular}

${ }^{a}$ The fractional pulsation upper limit to the $99 \%$ confidence level calculated according to Vaughan et al. 1994.

${ }^{\mathrm{b}}$ From combined MOS1 and MOS2 data.

${ }^{\mathrm{c}}$ Calculated from the FFT of two separate intervals of $\sim 3$ days of data with gaps.

measure (DM) of $337 \mathrm{pc} \mathrm{cm}^{-3}$ yields an estimated distance of $4.3 \mathrm{kpc}$ using the revised DM-distance model of Cordes \& Lazio (2002).

\section{DISCUSSION}

The position of 1WGA J1713.4-3949 determined from the $X M M$ data is R.A. $=17^{\mathrm{h}} 13^{\mathrm{m}} 28.4$, decl. $=-39^{\circ} 49^{\prime} 54^{\prime \prime} .5$ (J2000) (with an uncertainty of $\sim 6^{\prime \prime}$ ). Using our $20 \mathrm{~cm}$ radio data of SNR G347.3-0.5 obtained with Australia Telescope Compact Array (Lazendic et al. 2003), we found no radio counterpart for 1WGA J1713.4-3949 and derive a $5 \sigma$ upper limit for a point-source flux of $3 \mathrm{mJy}$. This is similar to the limit derived toward the CCO in Puppis A (Gaensler, Bock, \& Stappers 2000). There is no apparent optical emission at the location of 1WGA J1713.4-3949 in the Digitized Sky Survey ${ }^{5}$ image, which has an upper limit of $V>17 \mathrm{mag}$.

While the distance to the radio pulsar PSR J1713-3945 is broadly consistent with the SNR distance of $6 \pm 1 \mathrm{kpc}$ estimated by Slane et al. (1999), the pulsar is likely to be quite old $\left(\tau_{c} \sim 1.1 \mathrm{Myr}\right.$, compared with $\tau<40 \mathrm{kyr}$ for SNR G347.3-0.5) and does not have sufficient spin-down luminosity $\left(\dot{E} \sim 3.7 \times 10^{33} \mathrm{ergs} \mathrm{s}^{-1}\right)$ to power the observed X-ray flux from 1WGA J1713.4-3949 $\left(L_{\mathrm{X}} \sim 6 \times 10^{34} \mathrm{ergs} \mathrm{s}^{-1}\right)$. The newly determined radio timing position of the pulsar is $\sim 4^{\prime}$ in declination away from the position of 1WGA J1713.4-3949 (see Fig. 1). PSR J1713-3945 is therefore spatially coincident

\footnotetext{
${ }^{5}$ The Digitized Sky Survey was produced at the Space Telescope Science Institute under US Government grant NAG W-2166.
}

TABLE 3

Properties of PSR J1713-3945 Detected toward G347.3-0.5

\begin{tabular}{|c|c|}
\hline Parameter & Value $^{\mathrm{a}}$ \\
\hline R.A. (J2000) . & $17^{\mathrm{h}} 13^{\mathrm{m}} 14.23(3)$ \\
\hline Decl. (J2000) .. & $-39^{\circ} 45^{\prime} 47^{\prime \prime} .5(14)$ \\
\hline Period, $P(\mathrm{~ms}) \ldots \ldots \ldots \ldots \ldots$ & $392.451769234(8)$ \\
\hline Period derivative, $\dot{P}\left(\times 10^{-15}\right)$. & $5.7380(5)$ \\
\hline Dispersion measure, DM $\left(\mathrm{pc} \mathrm{cm}^{-3}\right)$. & $337(3)$ \\
\hline Epoch of period (MJD) .............. & 52165.0 \\
\hline rms residual $(\mathrm{ms})$........... & 1.1 \\
\hline Number of TOAs ...................... & 27 \\
\hline Timing span (days) ......... & 1200 \\
\hline Characteristic age, ${ }^{\mathrm{b}} \tau_{c}$ (Myr) $\ldots \ldots \ldots \ldots$ & 1.08 \\
\hline Surface magnetic field, ${ }^{\mathrm{c}} B\left(\times 10^{12} \mathrm{G}\right)$ & 1.52 \\
\hline Spin-down luminosity, ${ }^{\mathrm{d}} \dot{E}\left(\mathrm{ergs} \mathrm{s}^{-1}\right) \ldots$ & $3.74 \times 10^{33}$ \\
\hline
\end{tabular}

${ }^{a}$ Values in parentheses represent the $1 \sigma$ uncertainty in the least significant digit quoted.

${ }^{\mathrm{b}} \tau_{c} \equiv P / 2 \dot{P}$.

${ }^{\mathrm{c}} B \equiv 3.2 \times 10^{19}(P P)^{1 / 2} \mathrm{G}$, where $P$ is in seconds

${ }^{\mathrm{d}} \dot{E} \equiv 4 \pi^{2} I \dot{P} / P^{3}$, with $I=10^{45} \mathrm{~g} \mathrm{~cm}^{2}$ assumed. 
with SNR G347.3 -0.5 by chance, and there is likely no physical association between the two systems. We did not detect any X-ray source at the position of PSR J1713-3945 in Chandra or $X M M$ data. Adopting $N_{\mathrm{H}} \sim 5 \times 10^{21} \mathrm{~cm}^{-3}$ (by scaling the value for the SNR to the $4.3 \mathrm{kpc}$ distance of this pulsar), we derive an upper limit on the luminosity in the $0.5-10.0 \mathrm{keV}$ band of $2 \times 10^{31} \mathrm{ergs} \mathrm{s}^{-1}$ for nonthermal emission with a photon index of $\sim 1.5$ and an upper limit on the temperature of $82 \mathrm{eV}$ for blackbody emission from a $10 \mathrm{~km}$ radius NS.

The lack of radio and optical counterparts for 1WGA J1713.4-3949, the absence of X-ray pulsations with the current sensitivity and time resolution, the two-component spectrum and its associated luminosity are properties consistent with CCOs (Pavlov et al. 2002).

If this $\mathrm{CCO}$ is an accretion-powered NS, we can consider two scenarios: accretion from a fallback disk or accretion from a low-mass companion. The fallback disk has been suggested to form from residual material after the SNR explosion (e.g., Chevalier 1989) and has been used to explain and unify the properties of various types of NSs (Alpar 2001). However, limits derived from optical and infrared observations seem to disfavor this model, at least for some types of NSs (e.g., Kaplan et al. 2002). X-ray monitoring, in conjunction with optical and infrared limits, can be used to establish whether the NS is variable and thus likely to be accreting from a low-mass companion. Indeed, variability and a period of $6.4 \mathrm{hr}$ have been reported for one of the CCOs, J1617-5102 in SNR RCW 103, on which basis this CCO is claimed to be a binary (Sanwal et al. 2002). Comparing the unabsorbed X-ray flux of 1WGA J1713.4-3949 obtained from Chandra and XMM data for a blackbody fit with that of $A S C A$ data $\left[F_{\mathrm{X}}^{0}(0.5-10.0 \mathrm{keV})=\right.$ $5.3 \times 10^{-12} \mathrm{ergs}^{-2} \mathrm{~s}^{-1}$; Slane et al. 1999], we find no longterm $(\sim 3 \mathrm{yr})$ variability in 1WGA J1713.4-3949.

Interpretations of the emission from CCOs as thermal radiation from the surface of an NS run into problems because of high surface temperatures and small emitting areas derived from the blackbody model fits. The temperature we derived from the blackbody model of $0.4 \mathrm{keV}$ is much higher than the temperature predicted by the standard cooling model (Page \& Sarmiento 1996), while the temperature derived from the NSA models $(0.2 \mathrm{keV})$ would imply an NS age of $100-1000 \mathrm{yr}$, which is less than the estimated 20-40 kyr age of the SNR (Slane et al. 1999).

In the case in which CCOs are rotation-powered NSs, the nondetection of radio pulsations can be explained by an unfavorable beaming orientation. One of the objects, $1 \mathrm{E}$ 1207.4-5209 in SNR G296.5+10.0, that was originally identified as a CCO has been found to have a period of $424 \mathrm{~ms}$ with a faint pulsed fraction (7.6\%; Zavlin et al. 2000). Our limits on the pulsed fraction from 1WGA J1713.4-3949 are consistent with those derived toward other CCOs (7\%-15\%; e.g., Pavlov et al. 2002). Our limits indicate that 1WGA J1713.4-3949 is probably not an anomalous X-ray pulsar because anomalous X-ray pulsars are generally found to have spin periods between 6 and $12 \mathrm{~s}$ and pulse fractions of 10\%-70\% (e.g., Mereghetti 1998).

In summary, the nature of the compact central object in SNR G347.3-0.5 remains uncertain. Future observations are planned to help determine the exact nature of this source. Pointed Chandra observations are needed to provide a more accurate position and to enable a search for an optical counterpart, while deeper timing observations can search for periods substantially shorter than available from the current data.

We thank Peter Woods for useful discussions and Slava Zavlin for providing the files for the magnetic neutron star atmosphere. This work was supported in part by NASA contract NAS8-39073 (P. O. S.), grant GO0-1123X (J. S. L.), and grant GO2-3080B (J. P. H.).

\section{REFERENCES}

Alpar, M. A. 2001, ApJ, 554, 1245

Chevalier, R. A. 1989, ApJ, 346, 847

Cordes, J. M., \& Lazio, T. J. M. 2002, preprint (astro-ph/0207156)

Crawford, F., Pivovaroff, M. J., Kaspi, V. M., \& Manchester, R. N. 2002, in ASP Conf. Ser. 271, Neutron Stars in Supernova Remnants, ed. P. O. Slane \& B. M. Gaenlser (San Francisco: ASP), 37

Gaensler, B. M., Bock, D. C.-J., \& Stappers, B. W. 2000, ApJ, 537, L35

Gänsicke, B. T., Braje, T. M., \& Romani, R. W. 2002, A\&A, 386, 1001

Kaplan, D. L., Kulkarni, S. R., Frail, D. A., \& van Kerkwijk, M. H. 2002, ApJ, 566, 378

Lazendic, J., Slane, P., Gaensler, B., Plucinsky, P., Hughes, J., \& Reynolds, S. 2003, ApJ, submitted

Mereghetti, S. 1998, Mem. Soc. Astron. Italiana, 69, 819

Page, D., \& Sarmiento, A. 1996, ApJ, 473, 1067

Pannuti, T. G., Allen, G. E., Houck, J. C., \& Sturner, S. J. 2003, ApJ, 593, 377

Pavlov, G. G., Sanwal, D., Garmire, G. P., \& Zavlin, V. E. 2002, in ASP Conf. Ser. 271, Neutron Stars in Supernova Remnants, ed. P. O. Slane \& B. M. Gaenlser (San Francisco: ASP), 247
Pavlov, G. G., Shibanov, Y. A., Zavlin, V. E., \& Meyer, R. D. 1995, in The Lives of the Neutron Stars, ed. M. A. Alpar, Ü. Kiziloğlu, \& J. van Paradijs (Dordrecht: Kluwer), 71

Pfeffermann, E., \& Aschenbach, B. 1996, in Röntgenstrahlung from the Universe, ed. H. U. Zimmermann, J. Trümper, \& H. Yorke (MPE Rep. 263; Garching: MPE), 267

Sanwal, D., Garmire, G. P., Garmire, A., Pavlov, G. G., \& Mignani, R. 2002, AAS Meeting, 200, 72.01

Slane, P., Gaensler, B. M., Dame, T. M., Hughes, J. P., Plucinsky, P. P., \& Green, A. 1999, ApJ, 525, 357

Standish, E. M. 1990, A\&A, 233, 252

Thompson, C. 2000, in ASP Conf. Ser. 202, Pulsar Astronomy-2000 and Beyond, ed. M. Kramer, N. Wex, \& N. Wielebinski (IAU Colloq. 177; San Francisco: ASP), 669

Turner, T. J., \& Pounds, K. A. 1989, MNRAS, 240, 833

Vaughan, B. A., et al. 1994, ApJ, 435, 362

Zavlin, V. E., Pavlov, G. G., Sanwal, D., \& Trümper, J. 2000, ApJ, 540, L25

Zavlin, V. E., Pavlov, G. G., \& Shibanov, Y. A. 1996, A\&A, 315, 141 